\title{
Phase turbulence in Rayleigh-Bénard convection
}

\author{
Hao-wen $\mathrm{Xi},{ }^{1}$ Xiao-jun $\mathrm{Li}^{2}{ }^{2}$ and J. D. Gunton ${ }^{3,4}$ \\ ${ }^{1}$ Department of Physics and Astronomy, Bowling Green State University, Bowling Green, Ohio 43403 \\ ${ }^{2}$ Department of Physics, University of Washington, Seattle, Washington 98195-1560 \\ ${ }^{3}$ Department of Physics, Lehigh University, Bethlehem, Pennsylvania 18015* \\ ${ }^{4}$ Instituto Mediterraneo de Estudios Avanzados (CSIC-UIB), Campus Universitat Illes Balears, E-07071 Palma de Mallorca, Spain
}

(Received 27 March 2000)

\begin{abstract}
We present a three-dimensional simulation of Rayleigh-Bénard convection in a large aspect ratio $\Gamma=60$ with stress-free boundaries for a fluid Prandtl number $\sigma=0.5$. We find that a spatiotemporal chaotic state (phase turbulence) emerges immediately above onset, which we investigate as a function of the reduced control parameter $\epsilon$. In particular we find that the correlation length for the vertical velocity field, the time averaged convective current, and the mean square vorticity have power law behaviors near onset, with exponents given by $-1 / 2,1$, and $5 / 2$ respectively. We also find that the time averaged vertical velocity and vertical vorticity fields have the same (disordered) spatial characteristics as the corresponding instantaneous patterns for these fields, and that there is no long-term phase correlation in the system. Finally, we present simple theoretical explanations for the time averaged convective current as a function of the control parameter, and for the fact that the time dependence of three global quantities (characterizing the dissipation of kinetic energy, the release of internal energy by buoyancy, and entropy flow) is essentially the same.

PACS number(s): 47.54.+r, 47.20.Lz, 47.20.Bp, 47.27.Te
\end{abstract}

\section{INTRODUCTION}

The classical problem of Rayleigh-Bénard convection has a long and rich history, with many important experimental, theoretical, and simulation results obtained only in the past decade $[1,2]$. In Rayleigh-Bénard convection, a thin fluid layer of thickness $d$, confined between two horizontal parallel plates, is heated from below. When the temperature difference reaches a critical value of $\Delta T_{c}$ at which the buoyancy force exceeds viscous and thermal dissipation, the fluid undergoes a transition from a spatially and temporally uniform conduction state to a two-dimensional convective parallel roll state with a characteristic length $\lambda_{c} \sim 2 d$. In general, the dynamics of a convective system depends on three parameters: the Rayleigh number $R$, which represents the relation between the buoyancy and dissipative effects; the Prandtl number $\sigma$ of the fluid, which represents the ratio between the kinematic viscosity and the thermal diffusivity; and the geometrical parameter of the container size and shape. Busse extensively studied the stability domain of twodimensional straight parallel rolls as a function of wave number $k$, and Rayleigh number $R$ for many Prandtl numbers $\sigma$ in a laterally infinite system. He calculated the stability boundaries now known as the "Busse Balloon" [3]. It is well known that in a laterally infinite system with rigid-rigid boundaries at the top and bottom plates, there exists a stable, time-independent straight parallel roll state near the onset of convection for all Prandtl numbers $\sigma$. However, in the case of free-free boundaries at sufficiently low Prandtl numbers $(\sigma<0.543)$, Zippelius and Siggia $[4,5]$ and Busse and Bolton [6] in the early 1980's discovered that parallel rolls are unstable with respect to the skewed-varicose instability immediately above onset. An interesting implication of their

\footnotetext{
*Permanent address.
}

results is that, since no particular horizontal wave vector $\vec{k}$ is chosen as an orientation for steady parallel rolls, all of them (whose magnitudes are $|\vec{k}| \sim k_{c}$, where $k_{c}$ is the onset wave number) have to compete near onset. Consequently, the convective state is chaotic in time at arbitrarily small Reynolds numbers, with an arbitrary orientation of the wave vectors in the isotropic plane of the fluid. For this latter reason, the phenomenon is termed phase turbulence. This transition to spatiotemporal chaos (STC) occurs at the critical Rayleigh number $R_{c}$, where the bifurcation from the conduction state to an ordered convection state usually takes place. Thus phase turbulence provides another example of a direct transition from a spatially uniform stationary state to STC [7]. Other cases include the Küppers-Lortz transition [8] in Rayleigh-Bénard convection, the Fréedericksz transition in liquid crystals $[9,10]$, and certain one-dimensional models of STC. Tribelsky [11] showed that such direct transitions to STC occur as a consequence of the existence of a Goldstone mode (and an associated, slowly decaying "Goldstone band") in the system. The Goldstone mode results from an additional continuous symmetry in the system beyond the usual translations of the space-time coordinates. The chaos observed in such cases may be interpreted as a dynamical analog of second order phase transitions, with the order parameter related to the amplitudes of turbulent modes. For this reason it has been called soft mode turbulence [10]. In the case of phase turbulence discussed here, the Goldstone band describes the slow relaxation of the longwavelength modes of the vertical component of the vorticity $\vec{\omega}$ (where $\vec{\omega} \equiv$ $\vec{\nabla} \times \vec{u}$ with $\vec{u}$ the local fluid velocity).

In this paper, we report the results of a three-dimensional numerical study of phase turbulence in a large aspect ratio Rayleigh-Bénard cell with free-free boundary conditions. This study is based on the Boussinesq approximation to the 
hydrodynamic equations, and is an extension of work presented in an earlier publication [7]. There are two aspects of this study that are worth noting. First, phase turbulence only occurs for large values of the aspect ratio, $\Gamma \equiv L / d$, where $L$ is the horizontal dimension and $d$ is the thickness of the fluid layer. If the aspect ratio is small (such as the case in early studies of the transition to temporal chaos in RayleighBénard convection), the spatial degrees of freedom are suppressed, and phase turbulence does not occur. Second, phase turbulence is an example of weak turbulence, a term sometimes used to describe spatiotemporal chaos in a weakly driven system, i.e., one for which a control parameter [in this case the reduced Rayleigh number $\left.\left(\Delta T-\Delta T_{c}\right) / \Delta T_{c}\right]$ is of order 1 or smaller. This weak turbulence differs from the fully developed, strong turbulence flow, in which the energy is distributed over a large range of spatial and temporal scales. The phase turbulence flow we discuss here has about $90 \%$ of its kinetic energy contained near the wave number $|\vec{k}| \sim k_{c}$.

The paper is organized as follows: In Sec. II we discuss the model, and give a brief discussion of our numerical techniques. In Sec. III, we present quantitative results characterizing the spatiotemporal chaotic patterns, including the power spectrums for the vertical velocity and vertical vorticity fields. We also show that the time averaged vertical velocity and vertical vorticity fields have the same disordered spatial characteristics as the corresponding instantaneous patterns for these fields. This implies that there is no long-term phase correlation between patterns.

In Sec. IV, we give a detailed analysis of various spacetime averaged global quantities as functions of the control parameter $\epsilon$. In particular we find that the correlation length for the vertical velocity field, the time averaged convective current, and the mean square vorticity have power law behaviors near onset, with exponents given by $-1 / 2,1$, and $5 / 2$, respectively. In Sec. V, we present results for the temporal behavior of three space averaged global quantities, which characterize the dissipation of kinetic energy, the release of internal energy due to buoyancy, and the flow of entropy, respectively, and the vertical velocity at two different positions. In Sec. VI, we provide a simple theory to explain the behavior of the time averaged convective current as a function of the control parameter and the fact that the three global quantities have almost identical temporal behaviors. In Sec. V we give a short discussion of our results.

\section{FORMULATION AND NUMERICAL PROCEDURES}

In our three-dimensional simulation, we assume that the fluid satisfies the Boussinesq approximation. In this approximation, the temperature dependence of the fluid parameters (such as kinematic viscosity and thermal diffusivity) is neglected, except for the thermal expansion effect responsible for buoyancy. In the energy balance equation the viscous dissipation term is also neglected in comparison with the conductive term. The dimensionless Boussinesq equations, which describe the evolution of the velocity field $\vec{u}$ $=(u, v, w)$ and the deviation of the temperature field $\theta$ from the conductive solution, can be written as

$$
\vec{\nabla} \cdot \vec{u}=0
$$

$$
\begin{gathered}
\partial \vec{u} / \partial t+(\vec{u} \cdot \vec{\nabla}) \vec{u}=-\vec{\nabla} p+\sigma \theta \vec{e}_{z}+\sigma \vec{\nabla}^{2} \vec{u}, \\
\partial \theta / \partial t+\vec{u} \cdot \vec{\nabla} \theta=\vec{\nabla}^{2} \theta+w R,
\end{gathered}
$$

where $p$ is pressure and $\vec{e}_{z}$ is a unit vector in the vertical $z$ direction. In Eqs. (1) -(3), length and time have been made dimensionless in terms of the thickness of fluid layer $d$, and the vertical thermal diffusion time $\tau_{d}=d^{2} / \kappa$, respectively. The velocity, pressure, and temperature have been rescaled by $d / \tau_{d}=\kappa / d, \rho\left(d / \tau_{d}\right)^{2}=\rho \kappa^{2} / d^{2}$, and $\Delta T / R=\kappa \nu / \alpha g d^{3}$, respectively. Here $\alpha$ is the coefficient of thermal expansion, $\kappa$ is the thermal diffusivity, $\nu$ is the kinematic viscosity, $\rho$ is the fluid density, and $g$ is the acceleration due to gravity. The dimensionless control parameters for the problem are the Rayleigh number $R$,

$$
R=\alpha \Delta T g d^{3} / \nu \kappa,
$$

and Prandtl number $\sigma$,

$$
\sigma=\nu / \kappa,
$$

where $\Delta T$ is the imposed temperature difference. In the idealized limit of a laterally infinite system with free-free boundary conditions, the critical Rayleigh number $R_{c}$ $=27 \pi^{4} / 4$ and the onset wave number $k_{c}=\pi / \sqrt{2}$. In this paper, the Prandtl number is chosen to be $\sigma=0.5$, with Rayleigh numbers in the range $0 \leqslant \epsilon \leqslant 0.5$, where $\epsilon=(R$ $\left.-R_{c}\right) / R_{c}$ is the reduced Rayleigh number. We use mesh points $\mathrm{N}_{x} \times \mathrm{N}_{y} \times \mathrm{N}_{z}=256 \times 256 \times 18$ and uniform grid size $\Delta x=\Delta y=60 / 256$, and $\Delta z=1 / 18$. The system sizes are $L_{x}$ $=N_{x} \Delta_{x}=L_{y}=N_{y} \Delta_{y}=60$ and $L_{z}=N_{z} \Delta_{z}=1$. Thus the aspect ratio of the system $\left(\Gamma=L_{x} / L_{z}=L_{y} / L_{z}\right)$ is $\Gamma=60$ in our simulation.

The well-known marker-and-cell (MAC) $[12,13]$ finitedifference technique is employed in our simulation. The MAC method uses pressure and velocity as the primary dependent variables, with the velocities, temperature, and pressure located at the staggered mesh points. The basic solution algorithm is accurate to first order in time and to second order in space for a uniform spatial mesh. The stress free (free-free) boundary conditions at the upper and lower surfaces $(\mathrm{z}=0,1)$ are

$$
w=\frac{\partial u}{\partial z}=\frac{\partial v}{\partial z}=0 .
$$

The boundary condition for the velocities at the sidewalls is chosen to be no slip (rigid), i.e., the normal velocity and the tangential velocity at the sidewalls are zero,

$$
\left.\vec{u}\right|_{B}=0
$$

where $B$ denotes the boundary of the sidewall. The boundary conditions for the temperature $\theta$ on the upper and lower walls $(\mathrm{z}=0,1)$ and at the sidewalls are given by

$$
\left.\theta\right|_{z=0,1}=\left.\frac{\partial \theta}{\partial n}\right|_{B}=0
$$

where $n$ is normal to the boundary of the sidewall. Thus the temperature is fixed on the upper and lower walls, while the 
sidewalls are insulating. The time step required for numerical stability is determined by a standard linear stability analysis. For given Rayleigh number $R$ and Prandtl number $\sigma \leqslant 1$, we obtain

$$
\Delta t \leqslant\left(\frac{1}{\sigma^{-1}+1}\right)\left(\frac{1}{\Delta^{-2}+\sqrt{0.5 R \sigma^{-1}+2\left(\sigma^{-1}-1\right)^{2} \Delta^{-4}}}\right),
$$

where $\Delta^{-2}=\Delta x^{-2}+\Delta y^{-2}+\Delta z^{-2}$. For $\Delta x=\Delta y=60 / 256$, $\Delta z=1 / 18, \quad \sigma=0.5$, and $R \approx R_{c}=27 \pi^{4} / 4$, we have $\Delta t=$ $3.1 \times 10^{-4}$

To check our code, we have performed various tests. Here we present two examples. In the first case, we reproduced the steady parallel roll state near onset, by numerically solving the time-dependent three-dimensional hydrodynamic equations. The system was chosen to have an aspect ratio of (60:60:1) and a Prandtl number of 5.0, with free-free boundary conditions. We compared our numerical results for the maximum vertical velocity $w_{\max }$ for several different values of the control parameter $\epsilon$, with the theoretical values given by Ref. [14]. Our results are in very good agreement, i.e., within $1.5 \%$ for $0<\epsilon<0.15$, with the differences increasing with increasing epsilon, as one would expect, since the theory begins to break down for larger $\epsilon$. In the second example, we checked our code by comparing results with the numerical work of Kirchartz and Oertel [15]. These authors numerically studied steady cellular convection using rigidrigid boundary conditions with a Rayleigh number of 4000 and a Prandtl number of 0.71 , for an aspect ratio of a 10:4:1 system. We used exactly the same system with rigid-rigid boundary conditions, with similar grid points (we used $84 \times 36 \times 18$; they used $81 \times 33 \times 17$ ). The agreement of our results with theirs for the vertical velocity $w(x, y=2, z$ $=0.5)$ as a function of $x$ is excellent [Fig. 8(b) in their paper].

\section{CHARACTERIZATION OF SPATIOTEMPORAL CHAOS}

We first discuss the structure factors, spatial correlation functions, and correlation lengths for the vertical velocity and vertical vorticity fields, respectively, for one value of the control parameter $\epsilon=0.1$. The detailed description of several space-time averaged global quantities as a function of the control parameter $\epsilon$ is presented in Sec. IV.

\section{A. Vertical velocity field}

Figure 1(a) shows a typical instantaneous image of the vertical velocity field at the middle plane of the cell $w(x, y, z=1 / 2, t)$ from the numerical simulation at $\epsilon=0.1$. Dark regions correspond to hot rising fluid and white regions to cold descending fluid. The apparently random pattern is comprised of patches of different locally orientation rolls and many defects. The number of defects and orientations of the rolls fluctuates both in time and space. Figure 1(b) shows the corresponding two-dimensional structure factor $S(\vec{k}, t)$ for the instantaneous image. (The structure factor is the power spectrum in Fourier space.) One of the interesting features is that the intensity of the spectrum $S(\vec{k}, t)$ appears to be iso-

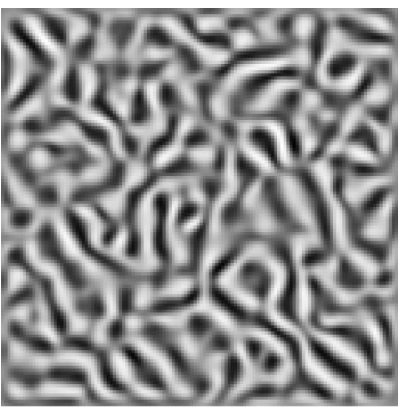

(a)

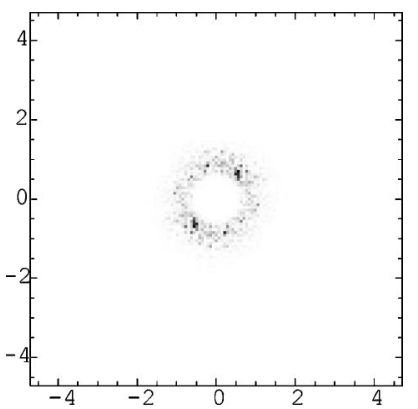

(b)
FIG. 1. (a) A typical instantaneous image of the vertical velocity field at the middle plane of the cell $w(x, y, z=1 / 2, t)$ with $\epsilon=0.1$. Dark regions correspond to hot rising fluid and white regions to cold descending fluid. (b) The corresponding structure factor $S(\vec{k}, t)$ for the instantaneous image, where the axes are $k_{x} / k_{c}$ and $k_{y} / k_{c}$. (The structure factor is the power spectrum in Fourier space.)

tropic azimuthally. Figure 2 shows the corresponding time averaged and azimuthally averaged power spectrum $S(k)$. We see that the power spectrum is skewed and peaked at a wave number $k_{\max }<k_{c}$.

In order to estimate the degree of spatial correlation in an instantaneous pattern, we also calculate the spatial autocorrelation function for the vertical velocity $w(x, y, z=1 / 2, t)$, which is defined as

$$
C(\vec{r}, t)=\left\langle w\left(\vec{r}^{\prime}, t\right) w\left(\vec{r}^{\prime}+\vec{r}, t\right)\right\rangle /\left\langle w\left(\vec{r}^{\prime}, t\right) w\left(\vec{r}^{\prime}, t\right)\right\rangle,
$$

where $\vec{r}=(x, y)$ and the bracket \langle\rangle denotes $\int d x^{\prime} d y^{\prime}$. [This spatial autocorrelation function is of course related to the structure factor in Fourier space by the relation $C(\vec{r}, t)$ $=\sum_{\vec{k}} S(\vec{k}, t) \exp (i \vec{k} \cdot \vec{r})$. These two methods are equivalent ways to analyze the spatial patterns.] The time averaged and azimuthally averaged spatial autocorrelation function $C(r)$ is shown in Fig. 3. This reveals a sharp central peak and a decaying oscillatory tail, and the peak-to-peak amplitude of the oscillatory tail can be approximated by a decaying exponential $\exp (-r / \xi)$. The correlation length $\xi \approx 2.36 d$ obtained from this fit is in reasonable agreement with estimates obtained from defining a correlation length from the variance of the power spectrum $S(k)$ in Fourier space, which is $\xi$

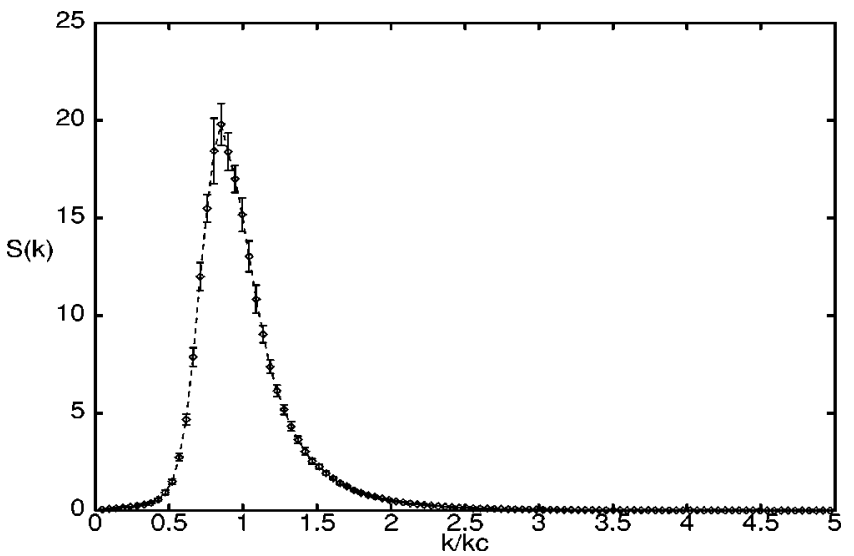

FIG. 2. The time averaged and azimuthally averaged power spectrum $S(k)$ at $\epsilon=0.1$. The dashed line is a guide to the eye. 


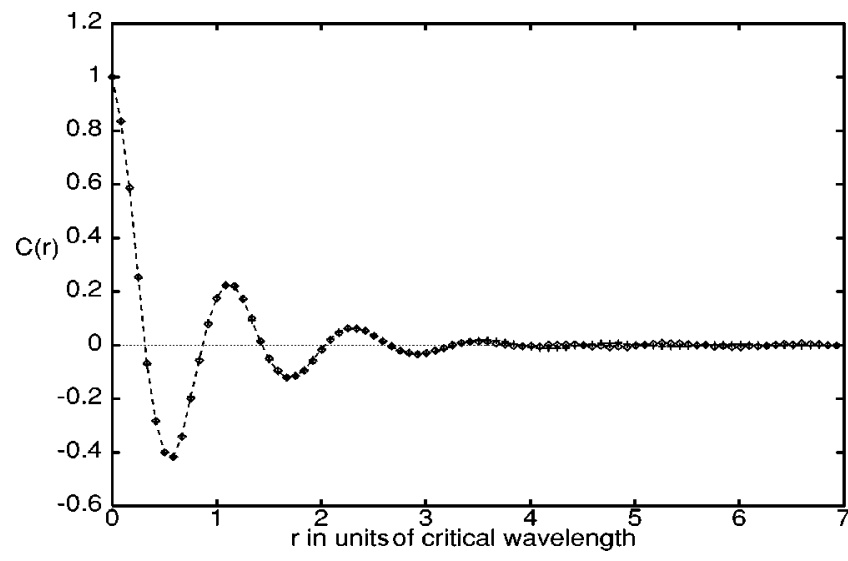

FIG. 3. The azimuthally averaged autocorrelation function $C(r)$ for the vertical velocity $w(x, y, z=1 / 2, t)$ at $\epsilon=0.1$. The diamonds are the time averaged autocorrelation function $C(r)$ of the instantaneous patterns; the pluses are the autocorrelation function $C(r)$ of the time averaged pattern. The dashed line is a guide to the eye.

$\approx 2.48 d$ [see Sec. IV C for details]. We also note that in our earlier work we found that near onset the time averaged structure factor satisfies a scaling behavior with respect to this correlation length, i.e., $k S(k) / \xi=F\left[\left(k-k_{\text {max }}\right) \xi\right]$. Here $F$ is the scaling function, and we have normalized the integral of $S(k)$ over $k$ space to be unity. Further details of these results for $S(k)$ and $\xi$ were given in our previous publication [7].

Another interesting issue is whether the structure of a time-averaged pattern is a featureless disordered state or has an inherent ordered symmetry. Although each instantaneous pattern is highly disordered, as shown in Fig. 1(a), the nature of the time-averaged pattern is not so obvious. Depending on whether or not there is a phase correlation between each pattern, the time-averaged pattern can either display a spatially periodic or spatially disordered pattern. For example, in the Faraday wave experiment, where a layer of fluid with a free surface is periodically driven in the direction normal to the surface, the time-averaged spatiotemporal chaotic images show strikingly regular structures [16]. In order to see whether the time averaged patterns show any spatial order in our system, we investigated the time averaged pattern for the vertical velocity field $\langle w(x, y, z=1 / 2)\rangle_{T}$,

$$
\langle w(x, y, z=1 / 2)\rangle_{T}=\frac{1}{T} \int_{0}^{T} w(x, y, z=1 / 2, t) d t,
$$

where the brackets \langle\rangle$_{T}$ denotes the time average. Qualitatively we observe that there is no significant difference between the time averaged pattern and a typical instantaneous pattern. Both patterns are highly disordered in real space and are approximately isotropic azimuthally in Fourier space. In order to study quantitatively the time averaged pattern, we calculated the spatial autocorrelation function for the time averaged pattern, and compared this with the time averaged autocorrelation function of the instantaneous patterns. Figure 3 shows the two azimuthally averaged autocorrelation functions. We observe that the two functions are almost identical. This suggests that the time averaged pattern has the same spatial characteristics as a typical instantaneous pattern, and that there is no long-term phase correlation in the system.

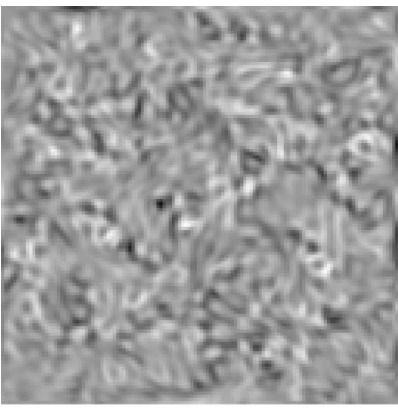

(a)

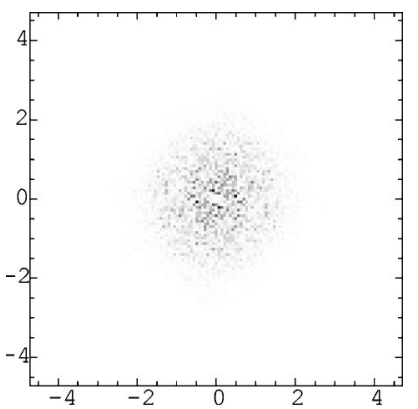

(b)
FIG. 4. (a) A disordered, instantaneous image of the vertical vorticity field at the middle plane of the cell $\omega_{z}(x, y, z=1 / 2, t)$, corresponding to Fig. 1(a). Dark and white regions indicate clockwise and counterclockwise rotations, respectively. (b) The corresponding two-dimensional structure factor $S_{\omega}(\vec{k}, t)$, where the axes are $k_{x} / k_{c}$ and $k_{y} / k_{c}$.

However, the maximum value of the time averaged velocity is much smaller than the value of each individual image.

\section{B. Vertical vorticity field}

Next, we analyze the patterns for the vertical vorticity field $\omega_{z}$, where $\omega_{z}=\partial_{x} v-\partial_{y} u$. Figure 4(a) shows a disordered, instantaneous image of the vertical vorticity field at the middle plane of the cell $\omega_{z}(x, y, z=1 / 2, t)$, corresponding to Fig. 1(a). Dark and white regions indicate clockwise and counterclockwise rotations, respectively. One observes that the vorticity field is interspersed with many defects, and that the defects fluctuate both in time and space. Figure 4(b) show the corresponding two-dimensional structure factor $S_{\omega}(\vec{k}, t)$, which reveals a broad azimuthally isotropic central peak and a decaying tail. Figure 5 shows the corresponding time averaged and azimuthally averaged power spectrum $S_{\omega}(k)$. We see that the power spectrum has a broad peak at small wave numbers $k$ and a decaying tail for large wave numbers $k$. The time averaged and azimuthally averaged spatial correlation $C_{\omega \omega}(r)$ is shown in Fig. 6. This shows a sharp localized central peak, and yields a decay length $\xi_{\omega}$ $\approx 0.85 d$ obtained from fitting to $\exp \left(-r / \xi_{\omega}\right)$. This value is again in good agreement with the value $\xi_{\omega}=0.848 \mathrm{~d}$ obtained from the variance of $S_{\omega}(k)$.

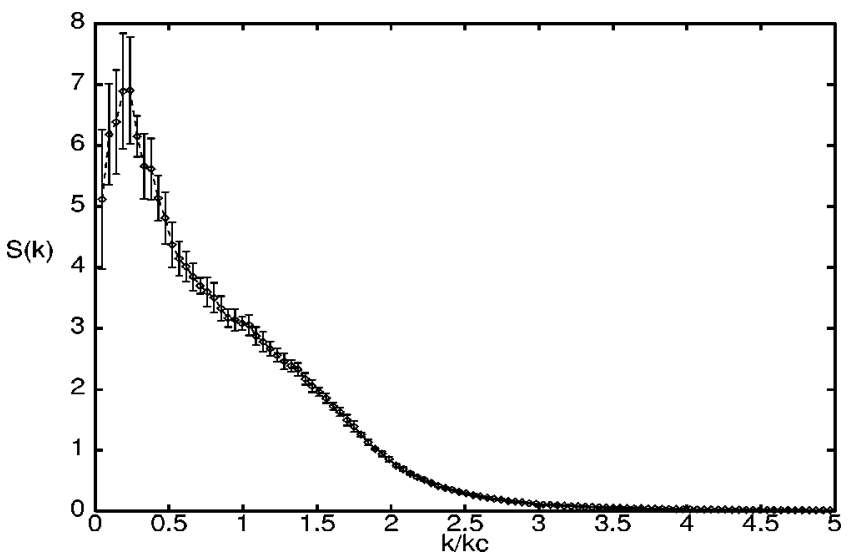

FIG. 5. The time averaged and azimuthally averaged power spectrum $S_{\omega}(k)$ at $\epsilon=0.1$. The dashed line is a guide to the eye. 


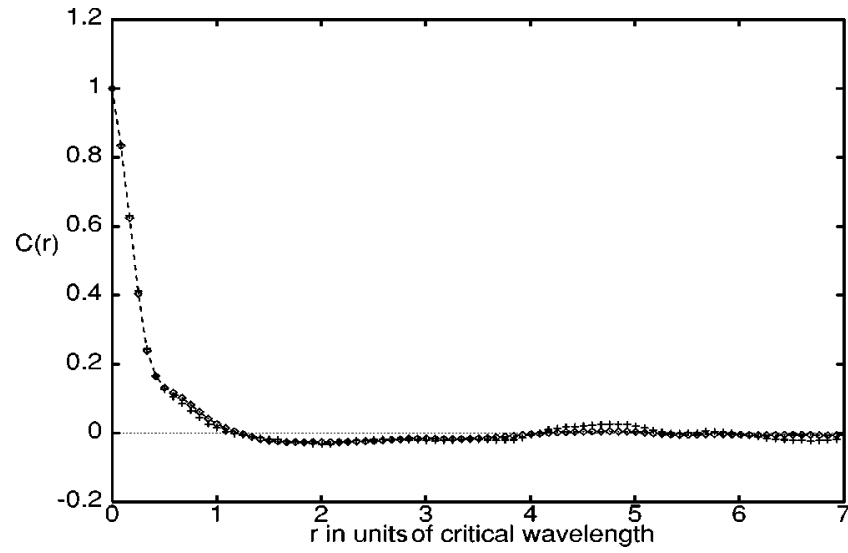

FIG. 6. The azimuthally averaged autocorrelation function $C_{\omega}(r)$ for the vertical vorticity $\omega(x, y, z=1 / 2, t)$ at $\epsilon=0.1$. The diamonds are the time averaged autocorrelation function $C_{\omega}(r)$ of the instantaneous patterns; the pluses are the autocorrelation function $C_{\omega}(r)$ of the time averaged pattern. The dashed line is a guide to the eye.

We have also investigated the time averaged pattern for the vertical vorticity field $\omega_{z}(x, y, z=1 / 2, t)$. As in the case of the vertical velocity pattern, we find that there is no significant difference between the time averaged pattern and a typical instantaneous pattern. Both the time averaged vertical vorticity image and each instantaneous image have the same random appearance in real space. We also calculated the spatial autocorrelation function for the time averaged pattern, and compared it with the time averaged autocorrelation function we obtained above. Both the azimuthally averaged correlation function for the time averaged image and the corresponding time averaged and azimuthally averaged correlation function for the instantaneous images are shown in Fig. 6. We find that the two functions are almost identical, as we found for the vertical velocity.

\section{SPACE AND TIME AVERAGED QUANTITIES AS FUNCTIONS OF THE CONTROL PARAMETER}

In this section, we investigate various space and time averaged global quantities as a function of the control parameter. In particular, we examine the Nusselt number $\mathrm{Nu}$, the spatial correlation lengths for the vertical velocity and vertical vorticity fields, and the mean square vorticity, $\Omega$, as a function of $\epsilon$.

\section{A. Nusselt number}

The Nusselt number $\mathrm{Nu}=1+\langle w \theta\rangle / R$ is the ratio of heat transport with and without convection, and is closely related to the conversion of potential energy into kinetic energy associated with the transfer of heat $\sigma\langle w \theta\rangle$. Here brackets \langle\rangle $=\int d x d y d z / V$ denote a volume averaging over the entire system. The convective current $J$ of the system is given by $(\mathrm{Nu}-1)$. It is well known that for a steady parallel roll state, the convective current increases linearly with $\epsilon / g_{2}$ near the onset of convection, where $g_{2}=0.5$ for free-free boundary conditions. We find that the time averaged convective current in phase turbulence is consistent with a linear relation, i.e., $\langle(N u-1)\rangle_{T}=\epsilon / g_{P T}$. However, we have $g_{P T}=1.27$

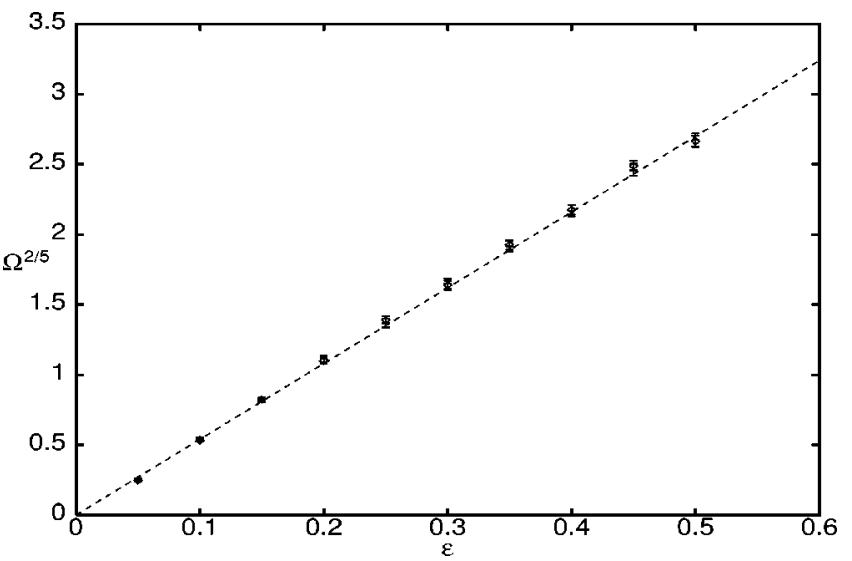

FIG. 7. The mean square vorticities $\Omega_{2 D}^{2 / 5}$ vs $\epsilon$ (diamond) and $\Omega_{3 D}^{2 / 5}$ vs $\epsilon$ (plus). The dash-dotted line is the fitting form of $\Omega^{2 / 5}$ $=5.47 \epsilon$.

\pm 0.03 , which is much larger than that of a parallel roll state. A theoretical explanation for this behavior is given in Sec. VI. Near the onset of convection the Nusselt number is also closely related to the vertical velocity field, since $(\mathrm{Nu}-1)$ $\propto \iint[w(x, y, z=1 / 2, t)]^{2} d x d y / A$. Thus we have $\langle w(x, y, z$ $\left.=1 / 2, t)^{2}\right\rangle \propto \epsilon$, (where \langle\rangle denotes $\left.(1 / T) \int d t(1 / A) \int d x d y\right)$.

\section{B. Mean square vorticity}

We now investigate another global quantity, related to the vertical vorticity field, as a function of $\epsilon$. We define this quantity $\Omega_{2 D}$ and $\Omega_{3 D}$ in two and and three dimensions as

$$
\begin{aligned}
& \Omega_{2 D}(t)=\frac{1}{A} \iint \omega_{z}^{2}(x, y, z=1 / 2, t) d x d y, \\
& \Omega_{3 D}(t)=\frac{1}{V} \iiint \omega_{z}^{2}(x, y, z, t) d x d y d z,
\end{aligned}
$$

respectively, where $\omega_{z}=(\vec{\nabla} \times \vec{u})_{z}=\partial_{x} v-\partial_{y} u$. Here $A$ and $V$ are the horizontal area and the volume of the Bénard cell, respectively. Clearly, $\Omega_{2 D}=\Omega_{3 D} \equiv 0$ in a parallel roll state, since $u(x, y, z, t)=u(x, z, t), v(x, y, z, t)=0$, and $w(x, y, z, t)$ $=w(x, z, t) . \Omega_{2 D}(x, y, z=1 / 2, t)$ is reminiscent of a Kolmogorov energy formulation in two-dimensional flow systems [17], in which $\Omega$ acts as a "vorticity energy." This mean square vorticity may also serve as an order parameter which characterizes the transition from an ordered two-dimensional parallel roll state to a spatiotemporal chaotic state. These functions can both be fit by a power law behavior, given by $\epsilon^{5 / 2}$. The resulting straight lines of the best power-law fit $\Omega_{2 D}^{2 / 5}$ vs $\epsilon$ and $\Omega_{3 D}^{2 / 5}$ vs $\epsilon$ are shown in Fig. 7. From Fig. 7, we note that the results for $\Omega_{2 D}$ and $\Omega_{3 D}$ are virtually the same. This suggests that the vertical vorticity $\omega_{z}(x, y, z, t)$ is independent of the vertical variable $z$. This is exactly what one would expect, since Zippelius and Siggia [4,5] showed that the leading contribution to the vertical vorticity is independent of z. However, a theoretical explanation of the power law behavior $\epsilon^{5 / 2}$ is much more complicated and will be presented elsewhere [18]. 


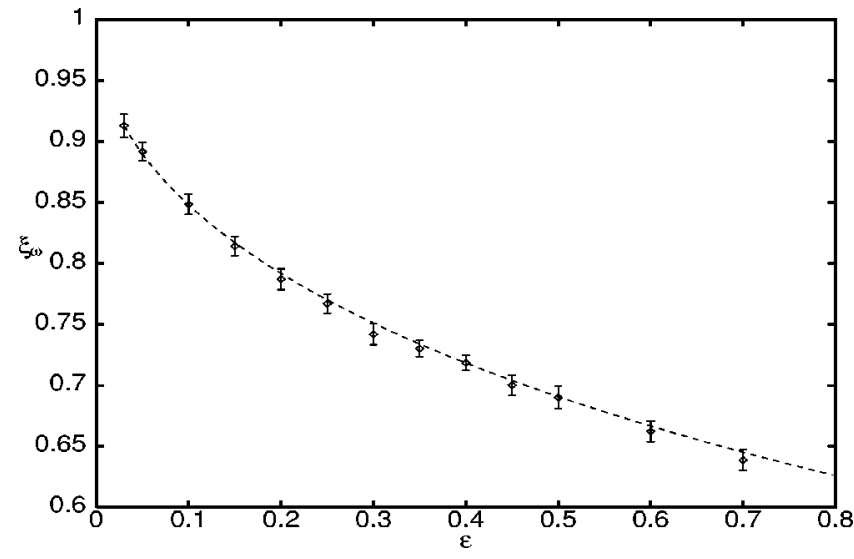

FIG. 8. The correlation length $\xi_{\omega}$ of the vertical vorticity field $\omega_{z}(x, y, z=1 / 2, t)$ vs $\epsilon$. The dashed line is a guide to the eye.

\section{Correlation lengths}

Spatiotemporal chaos generally results from a breakdown of global spatial coherence. However, a macroscopic coherence length - a length scale below which the pattern appears coherent-may still exist. Here we study two such lengths, namely, the spatial correlation lengths of the vertical velocity $w(x, y, z=1 / 2, t)$ and vertical vorticity $\omega_{z}(x, y, z=1 / 2, t)$, as functions of the control parameter $\epsilon$. We define a correlation length $\xi$ via the variance of the structure factor as

$$
\xi=\left(\left\langle k^{2}\right\rangle-\langle k\rangle^{2}\right)^{-1 / 2},
$$

where $\langle k\rangle$ and $\left\langle k^{2}\right\rangle$ are defined as

$$
\begin{aligned}
&\langle k\rangle= \frac{\int S(\vec{k}, t)|\vec{k}| d^{2} \vec{k}}{\int S(\vec{k}, t) d^{2} \vec{k}}, \\
&\left\langle k^{2}\right\rangle=\frac{\int S(\vec{k}, t)|\vec{k}|^{2} d^{2} \vec{k}}{\int S(\vec{k}, t) d^{2} \vec{k}} .
\end{aligned}
$$

We first investigate the correlation length for the vertical velocity field. Assuming a power law behavior of $\xi=\xi_{0}(\epsilon$ $\left.-\epsilon_{c}\right)^{-\nu}$, we find that the best fits are, $\nu=0.472 \pm 0.016, \xi_{0}$ $=0.82 \pm 0.04$, and $\epsilon_{c}=0.005$ [7]. The nonzero value $\epsilon_{c}$ can probably be attributed to the finite size of the system. The behavior of the correlation length is also consistent with a mean field power law exponent of $\nu=0.5$ and $\xi_{0}=0.78$. Note that the value of $\xi_{0}$ is a factor of $3 / 2$ larger than the value $\xi_{0}=\sqrt{8 / 3 \pi^{2}}=0.52$, as calculated from the curvature of the marginal stability curve.

While the correlation length of the vertical velocity field $w(x, y, z=1 / 2, t)$ diverges near onset as $\xi \sim \epsilon^{-1 / 2}$, suggesting a supercritical transition from the conduction state to the convective state, the behavior of the correlation length of the vertical vorticity field $\omega_{z}(x, y, z=1 / 2, t)$ turns out to be quite difficult to obtain. Using the same definition via the variance of the structure factor of the vorticity field as above, we calculate and plot the correlation length $\xi_{\omega}$ as a function of $\epsilon$ in Fig. 8. We find that this correlation length does not appear to obey a simple power law behavior, as $\xi$ does. More data close to $\epsilon=0$ are needed to reveal its asymptotic behavior.

\section{TEMPORAL BEHAVIOR}

\section{A. Global variables}

Before presenting the results of our simulation of the temporal behavior, we first consider some general hydrodynamic and thermodynamic relations which prove useful for a description of Rayleigh-Bénard convection. We take the scalar product of $\vec{u}$, and both sides of the momentum equation [Eq. (2)], and multiply both sides of the energy equation [Eq. (3)] by $\theta$. We then add the resulting equations term by term and integrate over the whole system. Taking into account the boundary conditions as well as the incompressibility condition, we obtain the evolution equation

$$
\frac{d}{d t} K(t)=\left[F_{1}(t)-F_{2}(t)\right]-\left[F_{3}(t)-F_{4}(t)\right]
$$

where $K(t)$ is defined as $K(t)=\frac{1}{2}\left\langle\vec{u} \cdot \vec{u}+\theta^{2}\right\rangle$, and the brackets \langle\rangle$=(1 / V) \int d x d y d z$ denote a spatial average over the entire volume. We note next that the dynamics of the global quantity $K(t)$ involves both dissipative and convective effects. Each of these contributions contains an irreversible part: (a) $F_{1}=\frac{1}{2} \sigma\left\langle\left(\partial u_{i} / \partial x_{j}+\partial u_{j} / \partial x_{i}\right)^{2}\right\rangle$ corresponds to the kinetic energy dissipated by viscosity. Here $i$ and $j$ are dummy indices over which a summation is performed and $u_{i}=(u, v, w), x_{j}=(x, y, z)$ (b) $F_{2}=\sigma\langle w \theta\rangle$ is the internal energy released by the buoyancy force. (c) $F_{3}=\langle\vec{\nabla} \theta \cdot \vec{\nabla} \theta\rangle$ corresponds to the dissipative thermal energy, i.e., generation of entropy due to temperature fluctuations. (d) $F_{4}=R\langle w \theta\rangle$ $=R F_{2} / \sigma$ represents the entropy flow due to the vertical velocity.

In the special case of a stationary state, one recovers the conditions $F_{1}=F_{2}$ and $F_{4}=F_{3}$, which are expressions of the balance between two competing mechanisms. It is worth noting that the Nusselt number $\mathrm{Nu}$ defined earlier can be expressed in terms of $F_{2}$ as $\mathrm{Nu}=1+F_{2} /(\sigma R)$. Also, $F_{2}$ and $F_{4}$ are not independent quantities, since $F_{4}=R F_{2} / \sigma$. Our main purpose in introducing these global quantities is that they provide us with a simple description of "energy balance" in Rayleigh-Bénard convection, and can be used to characterize the temporal dynamics.

It is convenient to rescale these quantities, with $F_{1}$ $=\sigma R_{c} F_{1}^{\prime}, F_{2}=\sigma R_{c} F_{2}^{\prime}, F_{3}=R R_{c} F_{3}^{\prime}$, and $F_{4}=R R_{c} F_{4}^{\prime}$, so that we have $F_{1}^{\prime}=F_{2}^{\prime}=F_{3}^{\prime}=F_{4}^{\prime}$ in a steady state. We showed representative time series of the quantities, $F_{1}^{\prime}(t), F_{2}^{\prime}(t)$, and $F_{3}^{\prime}(t)$ for $\epsilon=0.2$ in our previous publication [7]. The most important implication of that figure is the apparently chaotic behavior of these quantities over the time interval that is accessible to us. Indeed, in our earlier work we used the Grassberger-Procaccia method [19] to calculate the fractal dimensions $D_{f}$ for these quantities, and found $D_{f}=1.42$ +0.02 [7]. We also noted that the time dependence of these three quantities is almost exactly the same, i.e., $F_{1}^{\prime}(t)$ $=F_{2}^{\prime}(t)=F_{3}^{\prime}(t)$. In fact, this is the case for all the $\epsilon$ values studied in the range $0 \leqslant \epsilon \leqslant 0.5$. This is a surprising result considering the irregular spatiotemporal state we observed. This result also implies that the quantity $\mathfrak{R} \equiv \sigma F_{3} /\left(2 F_{2}\right.$ 


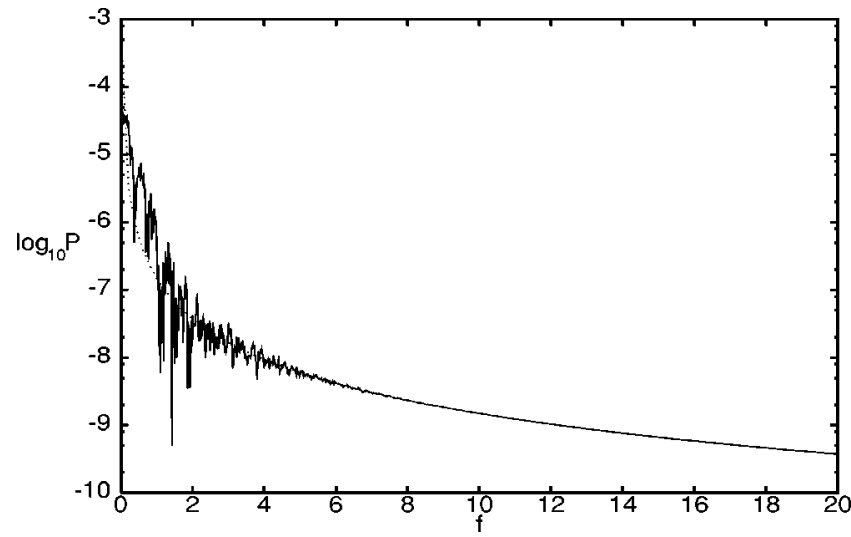

FIG. 9. The power spectrum of the global quantity $F_{1}^{\prime}(t)$ as a function of frequency $f$. The dashed line is the fitting form of $P(f) \sim f^{-2}$.

$\left.-F_{1}\right)=R F_{3}^{\prime} /\left(2 F_{2}^{\prime}-F_{1}^{\prime}\right)$, which is often used in a variational formulation to determine the critical Rayleigh number for the onset of convection, behaves as if the system is in a stationary state and $\mathfrak{R}=R$. This suggests that there are two attractors for a given $\epsilon$ (or $R$ ) near onset, one for the steady state, parallel roll case, and the other for the spatiotemporal chaotic state. In Sec. VI, we provide a theory to explain why $F_{1}^{\prime}(t)$ $=F_{2}^{\prime}(t)=F_{3}^{\prime}(t)$.

We conclude this section by noting that we have also calculated the Fourier spectrum of these global variables. Figure 9 shows this spectrum for the global variable $F_{1}^{\prime}(t)$. It is interesting to note that this spectrum behaves like $f^{-2}$ (cf. the dashed line) for large frequencies, which is characteristic of random Browian motion.

\section{B. Local variables}

It is also useful to consider the temporal behavior of local variables. In particular, we studied the local vertical velocity $w(x, y, z, t)$ as a function of time at two different spatial points. Figure 10 shows the time series for the vertical velocity $w\left(25,12 \frac{1}{2}, \frac{11}{12}\right)$. The power spectrum for this local variable is very different from that for the global variable, as can be seen in Fig. 11. At low frequencies it behaves like $\exp (-1.25 f)$, and at large frequencies has the same form of $f^{-2}$ as for the global variable.

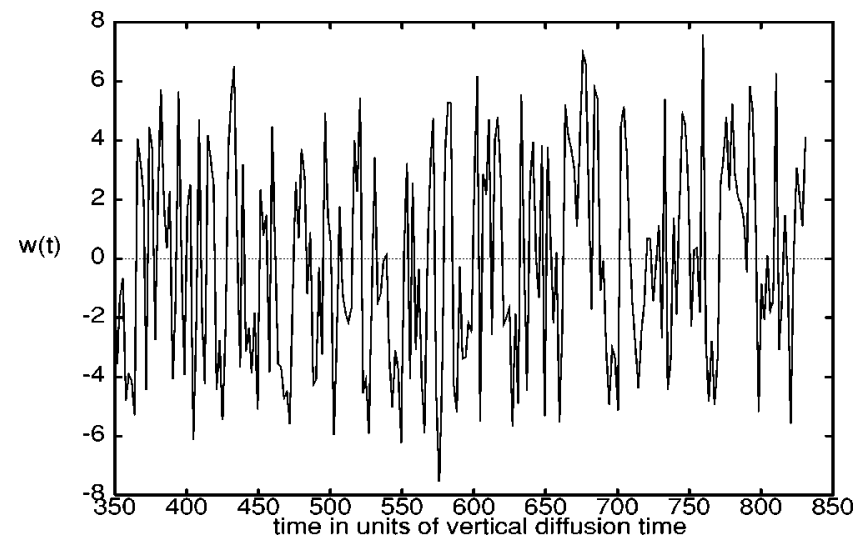

FIG. 10. Time series of the local vertical velocity $w\left(25,12 \frac{1}{2}, \frac{11}{12}, t\right)$.

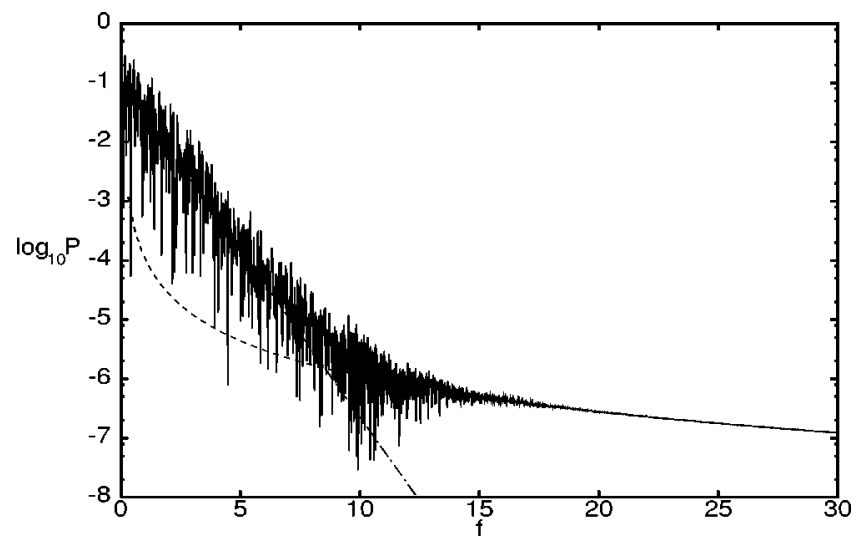

FIG. 11. The power spectra of the local vertical velocity $w\left(25,12 \frac{1}{2}, \frac{11}{12}, t\right)$ as a function of frequency $f$. The dashed lines are the fitting form of $P(f) \sim f^{-2}$ for large $f$, and the dash-dotted lines are $P(f) \sim \exp [-1.25 f]$ for small $f$.

\section{THEORY}

Finally, we present a simple theory to explain the fact that $F_{1}^{\prime}(t)=F_{2}^{\prime}(t)=F_{3}^{\prime}(t)$ and to evaluate the time averaged convective current, which satisfies $J=\epsilon / g_{P T}$, in phase turbulence. Our theory is based on the assumption that only those modes whose wave number is in the vicinity of $k_{c}$ are excited. Near onset, it is well known that the velocity field $\vec{u}$ $=\left(\vec{u}_{\perp}, w\right)=(u, v, w)$ and the temperature deviation $\theta$ can be approximated by order parameters $\psi(\vec{r}, t)$ and $\zeta(\vec{r}, t)$ in twodimensional space $\vec{r}$ multiplied by known, $z$-dependent prefactors. More precisely, one may take as an approximation that [20-22]

$$
\left[\vec{u}_{\perp}, w, \theta\right] \simeq\left[u_{0} \vec{\nabla}_{\perp} \psi+\vec{\nabla}_{\perp} \zeta(\vec{r}, t) \times \vec{e}_{z}, w_{0} \psi, \theta_{0} \psi\right],
$$

where $\vec{\nabla}_{\perp}$ is the gradient operator in two-dimensional space, and the prefactors for free-free boundary conditions (at $z$ $=0,1)$ are

$$
\begin{gathered}
u_{0}(z)=2 \sqrt{3} \cos (\pi z), \quad w_{0}(z)=\sqrt{3} \pi \sin (\pi z), \\
\theta_{0}(z)=\left(9 \sqrt{3} \pi^{3} / 2\right) \sin (\pi z) .
\end{gathered}
$$

With this approximation, it is straightforward to show that

$$
\begin{gathered}
F_{1}^{\prime}(t)=(2 / 9) O_{0}-\left(10 / 9 \pi^{2}\right) O_{1}+\left(8 / 9 \pi^{4}\right) O_{2}, \\
F_{2}^{\prime}(t)=O_{0}, \\
F_{3}^{\prime}(t)=(2 / 3) O_{0}-\left(2 / 3 \pi^{2}\right) O_{1},
\end{gathered}
$$

where we have applied $R \simeq R_{c}=27 \pi^{4} / 4$ and

$$
O_{n}=A^{-1} \int d \vec{r} \psi\left(\vec{\nabla}_{\perp}^{2}\right)^{n} \psi
$$

Now assume that only those modes which are in the vicinity of $k_{c}=\pi / \sqrt{2}$ are excited near onset. One may thus replace $\nabla_{\perp}^{2} \rightarrow-k_{c}^{2}$. This leads to

$$
F_{1}^{\prime}(t)=F_{2}^{\prime}(t)=F_{3}^{\prime}(t)=O_{0},
$$


in agreement with our numerical result. Note, however, that the power spectrum (structure factor) of the excited modes has a finite width. Consequently, these three quantities are not exactly the same.

Within the approximation of Eq. (19), the threedimensional hydrodynamical equations (1)-(3) can be approximated by the two-dimensional generalized SwiftHohenberg (GSH) model [20-22]. In the GSH model, the order parameter $\psi(\vec{r}, t)$ satisfies

$$
\tau_{0}\left[\partial \psi / \partial t+\vec{U} \cdot \vec{\nabla}_{\perp} \psi\right]=\left[\epsilon-\left(\xi_{0}^{2} / 4 k_{c}^{2}\right)\left(\vec{\nabla}_{\perp}^{2}+k_{c}^{2}\right)^{2}\right] \psi-N[\psi] .
$$

Here $\vec{U}(\vec{r}, t)$ is the mean flow velocity given by $\vec{U}(\vec{r}, t)$ $=\vec{\nabla}_{\perp} \zeta(\vec{r}, t) \times \vec{e}_{z}$, in which

$$
\left[\partial / \partial t-\sigma \vec{\nabla}_{\perp}^{2}\right] \vec{\nabla}_{\perp}^{2} \zeta=g_{m} \vec{e}_{z} \cdot\left[\vec{\nabla}_{\perp}\left(\vec{\nabla}_{\perp}^{2} \psi\right) \times \vec{\nabla}_{\perp} \psi\right] .
$$

The nonlinear $N[\psi]$ term has been evaluated in Fourier space at onset [21],

$$
N[\psi](\vec{k})=\sum_{\vec{k}_{2}, \vec{k}_{3}} g\left(\hat{k} \cdot \hat{k}_{2}\right) \hat{\psi}^{*}\left(\vec{k}_{2}, t\right) \hat{\psi}\left(\vec{k}_{3}, t\right) \hat{\psi}\left(\vec{k}+\vec{k}_{2}-\vec{k}_{3}, t\right),
$$

where $\hat{\psi}(\vec{k}, t)$ is the Fourier component of $\psi(\vec{r}, t)$ and the coupling constant $g(\cos \alpha)$ is given in Ref. [21] with $\alpha$ the angle between $\vec{k}$ and $\vec{k}_{2}$. For free-free boundary conditions, one has $\tau_{0}=2\left(1+\sigma^{-1}\right) / 3 \pi^{2}, \xi_{0}^{2}=8 / 3 \pi^{2}$, and $g_{m}=6$. The time averaged convective current $J=\langle\mathrm{Nu}\rangle_{T}-1$ is given by

$$
J=\frac{1}{A} \int d \vec{r}\left\langle\psi^{2}(\vec{r}, t)\right\rangle_{T}=\sum_{\vec{k}}\left\langle\hat{\psi}^{*}(\vec{k}, t) \hat{\psi}(\vec{k}, t)\right\rangle_{T},
$$

where \langle\rangle$_{T}=T^{-1} \int d t$ denotes time average. To calculate $J$, we multiply both sides of Eq. (25) by $\psi(\vec{r}, t)$ and then take the spatial and time averages. This leads to, in Fourier space,

$$
\begin{aligned}
\sum_{\vec{k}}[ & \left.\epsilon-\xi_{0}^{2}\left(k^{2}-k_{c}^{2}\right)^{2} / 4 k_{c}^{2}\right]\left\langle\hat{\psi}^{*}(\vec{k}, t) \hat{\psi}(\vec{k}, t)\right\rangle_{T} \\
& -\sum_{\vec{k}_{1}, \vec{k}_{2}, \vec{k}_{3}, \vec{k}_{4}} g\left(\hat{k}_{1} \cdot \hat{k}_{2}\right) \\
& \times\left\langle\hat{\psi}^{*}\left(\vec{k}_{1}, t\right) \hat{\psi}^{*}\left(\vec{k}_{2}, t\right) \hat{\psi}\left(\vec{k}_{3}, t\right) \hat{\psi}\left(\vec{k}_{4}, t\right)\right\rangle_{T} \delta_{\vec{k}_{1}+\vec{k}_{2}, \vec{k}_{3}+\vec{k}_{4}} \\
= & 0 .
\end{aligned}
$$

Note that the contribution from the $\vec{U} \cdot \vec{\nabla}_{\perp} \psi$ term in Eq. (25) can be converted into a surface term which vanishes under boundary conditions.

We now propose a simple model for phase turbulence. We assume that a phase turbulent state is composed of many parallel rolls, whose wave numbers lie on the ring of $|\vec{k}|$ $=k_{c}$, and whose amplitudes are all equal, but whose phases and whose orientations are random in time. More precisely, we assume that

$$
\hat{\psi}(\vec{k}, t)=\psi_{0}(t) \delta_{k, k_{c}} \sum_{i=1}^{M} e^{i \phi\left(\beta_{i}, t\right)} \delta_{\alpha, \beta_{i}(t)},
$$

where $\psi_{0}(t)$ and $\phi\left(\beta_{i}, t\right)$ are the amplitude and the phases of the selected modes, $k=k_{c}$ and $\beta_{i}(t)$ are the amplitude, and the angles of their corresponding wave numbers, while $M$ is the total number of the modes and $\alpha$ is the angle between $\vec{k}$ and some reference direction. Since $\mathrm{Nu}-1$ $=\Sigma_{\vec{k}}|\hat{\psi}(\vec{k}, t)|^{2}=M \psi_{0}^{2}(t)$ has a well-defined time averaged value with small fluctuations [7], we expect the same behavior for $\psi_{0}(t)$. But we speculate that both the phases $\phi\left(\beta_{i}, t\right)$ and the angular distribution $\left\{\beta_{i}(t)\right\}$ are random in time, which leads to the spatiotemporal chaotic behavior in phase turbulence. Note that this form of $\hat{\psi}(\vec{k}, t)$ is also consistent with the corresponding one for steady states. For example, one has $M=2, \psi_{0}=$ const, $\phi\left(\beta_{i}, t\right)=$ const, and $\beta_{i}=i \pi$ with $i=0$ and 1 for a parallel roll state, and $M=6, \psi_{0}=$ const, $\phi\left(\beta_{i}, t\right)=$ const, and $\beta_{i}=i \pi / 3$ with $i=0,1, \ldots, 5$ for a hexagonal state [21]. For phase turbulence, we take $M \rightarrow+\infty$.

We now use this model to calculate the time averaged convective current from Eq. (29). The first term can be easily evaluated as $\epsilon J$. Note that there are only three possibilities for the constraint $\vec{k}_{1}+\vec{k}_{2}=\vec{k}_{3}+\vec{k}_{4}$ in the second term to be satisfied on a ring: (a) If $\vec{k}_{1}+\vec{k}_{2}=0$, then $\vec{k}_{3}+\vec{k}_{4}=0$. (b) if $\vec{k}_{1} \neq \vec{k}_{2}$ and $\vec{k}_{1}+\vec{k}_{2} \neq 0$, then either $\vec{k}_{3}=\vec{k}_{1}$ and $\vec{k}_{4}=\vec{k}_{2}$ or $\vec{k}_{3}$ $=\vec{k}_{2}$ and $\vec{k}_{4}=\vec{k}_{1}$; or, (c) if $\vec{k}_{1}=\vec{k}_{2}$, then $\vec{k}_{3}=\vec{k}_{4}=\vec{k}_{1}$. It is more convenient to express these constraints in terms of their angles, which can be summarized, correspondingly, as (a) $\quad \delta_{\alpha_{2}, \alpha_{1}+\pi} \delta_{\alpha_{4}, \alpha_{3}+\pi}, \quad$ (b) $\quad\left(1-\delta_{\alpha_{2}, \alpha_{1}+\pi}-\right.$ $\left.\delta_{\alpha_{2}, \alpha_{1}}\right)\left(\delta_{\alpha_{3}, \alpha_{1}} \delta_{\alpha_{4}, \alpha_{2}}+\delta_{\alpha_{3}, \alpha_{2}} \delta_{\alpha_{4}, \alpha_{1}}\right), \quad$ and $\quad$ (c) $\delta_{\alpha_{2}, \alpha_{1}} \delta_{\alpha_{3}, \alpha_{1}} \delta_{\alpha_{4}, \alpha_{1}}$. Now inserting Eq. (30) into the second term of Eq. (29), and applying $\phi\left(\beta_{i}+\pi\right)=-\phi\left(\beta_{i}, t\right)$ [since $\left.\hat{\psi}^{*}(\vec{k}, t)=\hat{\psi}(-\vec{k}, t)\right]$ and these constraints, after some algebra one finds that the second term is simply $-M^{2} g_{M}\left\langle\psi_{0}^{4}(t)\right\rangle_{T}$ with

$$
\begin{aligned}
g_{M}= & g(-1)\left(1-\frac{2}{M}\right)-\frac{1}{M} g(1) \\
& +\frac{2}{M^{2}} \sum_{i, j=1}^{M}\left\langle g\left[\cos \left(\beta_{i}(t)-\beta_{j}(t)\right)\right]\right\rangle_{T} .
\end{aligned}
$$

Here we have decoupled $\left\langle\psi_{0}^{4}(t)\right\rangle_{T}$ and $\left\langle g\left[\cos \left(\beta_{i}(t)\right.\right.\right.$ $\left.\left.\left.-\beta_{j}(t)\right)\right]\right\rangle_{T}$. Since $J=M\left\langle\psi_{0}^{2}(t)\right\rangle_{T}$, if one neglects the fluctuations of $\psi_{0}(t)$, one has then $M^{2}\left\langle\psi^{4}(t)\right\rangle_{T} \simeq J^{2}$. From Eq. (29), this leads to the solution for the time averaged convective current

$$
J \simeq \epsilon / g_{M},
$$

in addition to the conduction solution $J=0$. This solution reproduces the known results [21] for both parallel rolls with $g_{2}=g(-1)+\frac{1}{2} g(1)$ and hexagons with $g_{6}=\frac{1}{6}[6 g(-1)$ $\left.+4 g\left(-\frac{1}{2}\right)+4 g\left(\frac{1}{2}\right)+g(1)\right]$. For phase turbulence, we expect that $\beta_{i}(t)-\beta_{j}(t)$ distributes uniformly between $[0,2 \pi]$. Taking $M \rightarrow \infty$ and replacing $\sum_{i=1}^{M} \rightarrow(M / 2 \pi) \int_{0}^{2 \pi} d \alpha$, one finds from Eq. (31) that 


$$
\begin{aligned}
g_{P T}= & g(-1)+\frac{2}{\pi} \int_{0}^{\pi} d \alpha g(\cos \alpha)=0.855922 \\
& +0.0458144 \sigma^{-1}+0.0709326 \sigma^{-2}
\end{aligned}
$$

where $\sigma$ is the Prandtl number, and the explicit formula for $g(\cos \alpha)$ is given in Ref. [21]. For $\sigma=0.5$ as in our study, this simple theory gives $J=\epsilon / g_{P T}$ with $g_{P T} \simeq 1.2313$, very close to our numerical result $g_{P T} \simeq 1.27 \pm 0.03$, as discussed in Sec. IV A. Considering all the approximations we have made, such a good agreement is very encouraging.

However, this simple model apparently misses two important features of phase turbulence [18]. The first is the lack of the mean square vorticity. Since all $\vec{k}$ is lie on a single ring in our model, the vorticity is identically zero. This, however, is not born out by our numerical calculations. Second, the structure factor from our numerical calculations has a finite width near its peak position, which leads to a significant reduction on the value of $J$. An improved theory will be presented elsewhere [18].

\section{SUMMARY}

In summary, we have presented a three-dimensional simulation of Rayleigh-Bénard convection in a large aspect ratio $\Gamma=60$ with stress-free boundaries for a fluid Prandtl number $\sigma=0.5$. We have investigated the spatiotemporal behavior as a function of the reduced control parameter $\epsilon$ near the onset of the second order transition. In particular,we calculated the spatial correlation functions and Fourier spectrums of the chaotic pattern as functions of the control parameter $\epsilon$. The spatial two-point correlation length for the vertical velocity field is consistent with $\xi \sim \epsilon^{-1 / 2}$, while the spatial correlation length for the vertical vorticity requires further investigation. We also found that the time averaged convective current $J$ and the time averaged vorticity current $\Omega$ have power law behaviors given by $J \sim \epsilon^{1 / 2}$ and $\Omega \sim \epsilon^{5 / 2}$. Finally, we presented an argument which predicts that three global quantities which can be used to characterize the energy balance in phase turbulence should have essentially the same time dependence near onset. We also explained the behavior of the time averaged convective current as a function of $\epsilon$. An improved version of this calculation which also predicts the behavior of the mean square vorticity as a function of $\epsilon$, will be presented elsewhere. Finally, we note that further study of the structure factor for the vertical vorticity for larger aspect ratios is necessary in order to obtain a better understanding of this function and its associated correlation length.

\section{ACKNOWLEDGMENTS}

This work was supported by the National Science Foundation under Grant No. DMR-9813409. H.W.X. was also supported by the Research Corporation under Grant No. CC4250. One of us (J.D.G.) wishes to acknowledge support from IBERDROLA during part of this project. X.J.L. also knowledges the support of the National Science Foundation under Grant No. DMR9876864 during the preparation of the manuscript. Numerical work reported here was carried out on the Cray-C90 at Pittsburgh Supercomputing Center and Cray-YMP8 at the Ohio Supercomputer Center.
[1] M.C. Cross and P.C. Hohenberg, Rev. Mod. Phys. 65, 3 (1993).

[2] G. Ahlers, in 25 Years of Nonequilibrium Statistical Mechanics, edited by J. J. Brey et al. (Springer, New York, 1995), p. 91.

[3] F.H. Busse, Rep. Prog. Phys. 41, 1929 (1978).

[4] A. Zippelius and E.D. Siggia, Phys. Rev. A 26, 1788 (1982).

[5] A. Zippelius and E.D. Siggia, Phys. Fluids 26, 2905 (1983).

[6] F.H. Busse and E.W. Bolton, J. Fluid Mech. 146, 115 (1984).

[7] Hao-wen Xi, Xiao-jun Li, and J.D. Gunton, Phys. Rev. Lett. 78, 1046 (1997).

[8] G. Küppers and D. Lortz, J. Fluid Mech. 35, 609 (1969); G. Küppers, Phys. Lett. A 32, 7 (1970).

[9] H. Richter, A. Buka, and I. Rehberg, Phys. Rev. E 51, 5886 (1995); H. Richter, N. Kloepper, A. Hertrich, and A. Buka, Europhys. Lett. 30, 37 (1995).

[10] S. Kai, K. Hayashi, and Y. Hidaka, J. Phys. Chem. 100, 19007 (1996).

[11] M.I. Tribelsky, Int. J. Bifurcation Chaos Appl. Sci. Eng. 7, 997 (1997); Phys. Usp. 40, 159 (1997).
[12] F.H. Harlow and J.E. Welch, Phys. Fluids 8, 2182 (1965).

[13] B. D. Nichols, C. W. Hirt, and R. S. Hotchkiss, Los Alamos Scientific Lab, Report No. LA-8355 (1989).

[14] A. Schlüter, D. Lortz, and F. Busse, J. Fluid Mech. 23, 129 (1965).

[15] K.R. Kirchartz and H. Oertel, Jr., J. Fluid Mech. 192, 249 (1988).

[16] B.J. Gluckman, C.B. Arnold, and J.P. Gollub, Phys. Rev. E 51, 1128 (1995).

[17] R.H. Kraichnan, Phys. Fluids 10, 1417 (1967); G.K. Batchelor, ibid. 12, 233 (1969).

[18] Xiao-jun Li, Hao-wen Xi, and J. D. Gunton (unpublished).

[19] P. Grassberger and I. Procaccia, Phys. Rev. Lett. 50, 346 (1983).

[20] J. Swift and P.C. Hohenberg, Phys. Rev. A 15, 319 (1977).

[21] M.C. Cross, Phys. Fluids 23, 1727 (1980).

[22] E.D. Siggia and A. Zippelius, Phys. Rev. Lett. 47, 835 (1981); M.C. Cross, Phys. Rev. A 27, 490 (1983); P. Mannevill, J. Phys. (Paris) 44, 759 (1983). 